\title{
Pathological Electromyogram (EMG) Signal Analysis Parameters
}

F. Meziani, S. Rerbal and S. M. Debbal ${ }^{*}$

Consultant Intervention Cardiologist Nepal Mediciti Hospital Lalitpur, Nepal

*Corresponding Author: M. Debbal, Genie Biomedical Laboratory (GBM) Faculty of Technology University A.B.Belkaid-Tlemcen BP 119 Tlemcen; Algeria

Received Date: May 17, 2021; Accepted Date: June 16, 2021; Published Date: June 21, 2021

Citation: Meziani, SF. Rerbal S and S. M. Debbal (2021 Pathological electromyogram (EMG) signal analysis parameters. J. Clinical Cardiology and Cardiovascular Interventions, 4(13); Doi:10.31579/2641-0419/185

Copyright: (C) 2021 M. Debbal, This is an open-access article distributed under the terms of the Creative Commons Attribution License, which permits unrestricted use, distribution, and reproduction in any medium, provided the original author and source are credited.

ABSTRACT
Clinical analysis of the electromyogram is a powerful tool for diagnosis of neuromuscular diseases. There fore,
the detection and the analysis of electromyogram signals has he attracted much attention over the years. Several
methods based on modern signal Processing techniques such as temporal analysis, spectro-temporel analysis ...,
have been investigated for electromyogram signal treatment. However, many of these analysis methods are not
highly successful due to their complexity and non-stationarity. The aim of this study is to analyse the EMGs
signals using nonlinear analysis. This analysis can provide a wide range of information's related to the type of
signal (normal and pathological
Key words: electromyogram; EMG; signal; nonlinear analysis; parameters; pathology

\section{Introduction:}

The EMG signal is a biomedical signal that measures electrical currents generated in muscles during its contraction representing neuromuscular activities. The nervous system always controls the muscle activity (contraction/relaxation). Hence, the EMG signal is a complicated signal, which is controlled by the nervous system and is dependent on the anatomical and physiological properties of muscles.

Detection and analysis of EMGs signals with powerful and advance methodologies is becoming a very important requirement in biomedical engineering. More recently, nonlinear tools have been introduced to analyze the EMGs signals; among them, the recurrence quantification analysis (RQA) and the bispectral analysis.

The recurrence quantification analysis (RQA) was shown to be particularly promising for the detection of muscle status changes [1]. The recurrence plots have been widely explored scientific fields for understanding the dynamics of system. Recurrence quantification analysis (RQA) methods for biological signals such as electroencephalogram [2] and EMG [3], are useful since it does not require large data and does not depend on statistical nature of signal [4].

In addition, many signals have nonlinearity and non-Gaussian behavior, and such signals cannot be examined properly by 2 nd-order statistical methods. Thus, higher-order statistical methods have been proved. The HOS was first applied to real signal processing problems in the 1970s and since then it has continued to be applied in many different areas, such as economics, speech signal processing, noisy and artifact removal, biomedical signal processing, and optics. Since EMG signals are nonstationary and non-Gaussian signals, they should be examined by HOS methods. Bispectrum, which is the Fourier transform of the 3rdorder cumulant, can be applied to nonlinear and non-Gaussian signals to extract nonlinear information. [5]
The electromyographic (EMG) signals are extensively used on feature extraction methods for movement classification purposes. High-order statistics (HOS) is being employed increasingly in myoelectric research. HOS techniques could be represented in the frequency domain (highorder spectra, e.g., bispectrum, trispectrum) or in the time domain (higherorder cumulants).

The objective of this article is to applied the non-linear analyze of the EMGs signals (normal, myopathy and neuropathy)

\section{Study of energy}

In the literature various approaches can be found to extract the EMG envelogram which will be useful in diagnosing various pathological cases. There are several methods to extract the envelope such as the calculation of the square of the signal or absolute value (equations (1-2)). The square of the samples of a given signal (equation (1)) makes it possible to evaluate its energy in the temporal field. However, and as illustrated in Figure.1, samples of high amplitude are very heavily favoured over those of low amplitude. The amplitude of the energy calculated by the absolute value [equation (2)] of the samples of the signal also disadvantages samples of low amplitude.

Two other approaches that can be used are Shannon entropy and Shannon energy; see equations (3) and (4). These approaches give greater weight to the average intensities of the signal; therefore, the noise of low intensity and high intensity of disturbance will be mitigated. Similarly, the Shannon entropy [equation (3)] does not yield the true proportions of the signal, attenuating more samples of very low amplitude for the benefit of large-amplitude oscillations. The Shannon energy [equation (4)] proves the median approach, making it possible to generate a representation that takes account of the physiological attenuation of sounds as well as artefacts of large amplitude while recording the EMG signal. The EMG 
signal energy representations [Figure.2] highlight the interest of the Shannon energy. According to these figures, we can see that only the Shannon entropy and the Shannon energy can absorb the magnitude of oscillations of high intensity as well as those in low amplitudes. So, they are the most suitable for the treatment of EMG signal.

- $\quad$ Square of the energy: $\mathrm{S}(\mathrm{t})^{2}$

$$
\mathrm{En}=
$$

(1)
- Absolute value of the energy : $\mathrm{V}=$ $|\mathrm{S}(\mathrm{t})|$

- $\quad$ Shannon entropy : $\quad \mathrm{Ep}=-|\mathrm{S}(\mathrm{t})| *$ $\log |\mathrm{S}(\mathrm{t})|$

- $\quad$ Shannon energy: $\quad \mathrm{Es}=-\mathrm{S}(\mathrm{t})^{2} *$ $\log \mathrm{S}(\mathrm{t})^{2}$

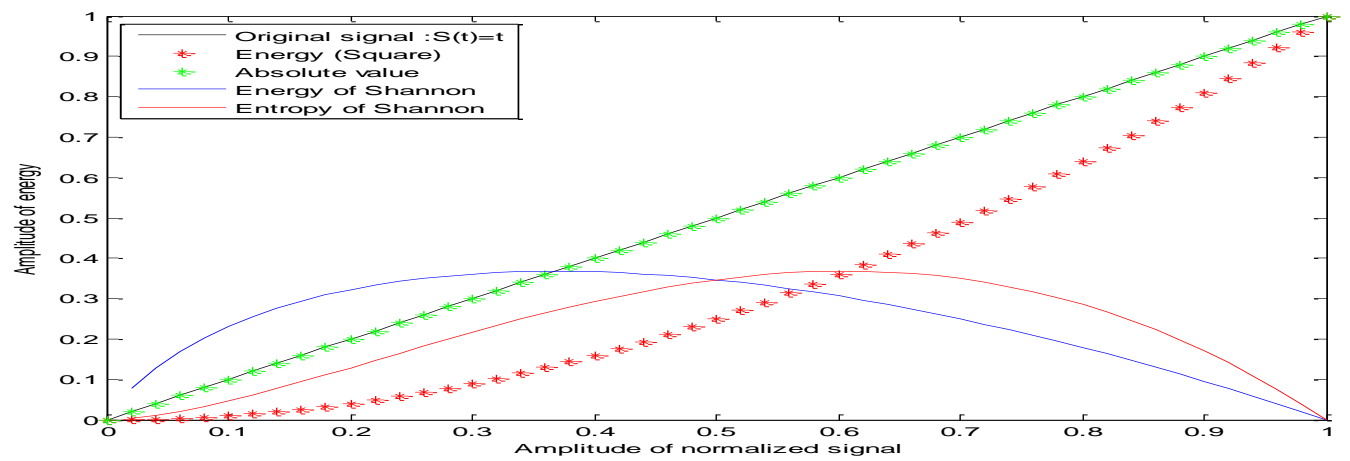

Figure 1: Temporal energy representation of the signal s (t).

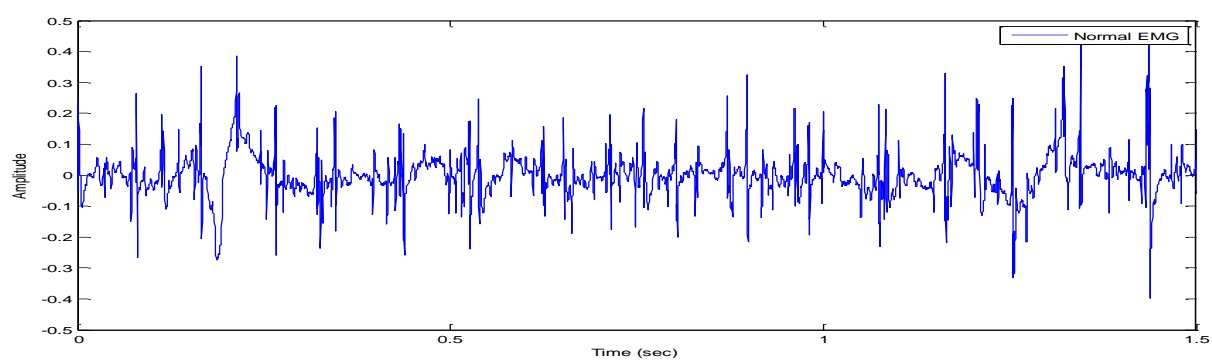

(a)

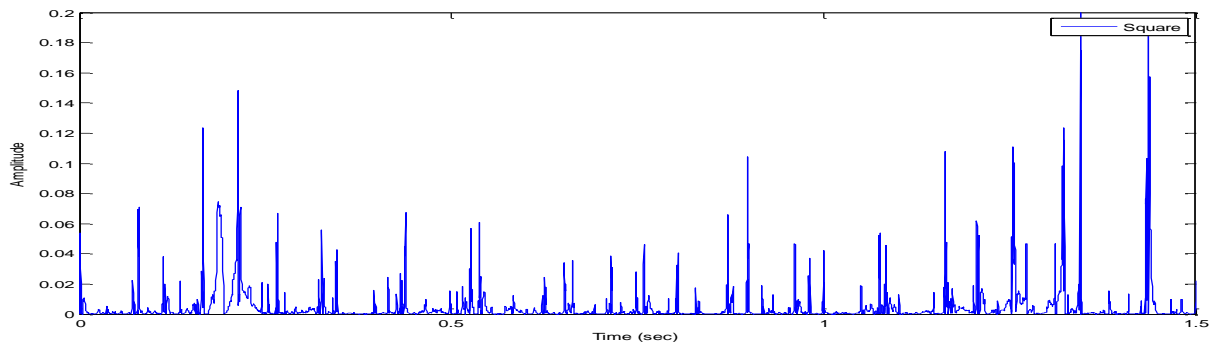

(b)

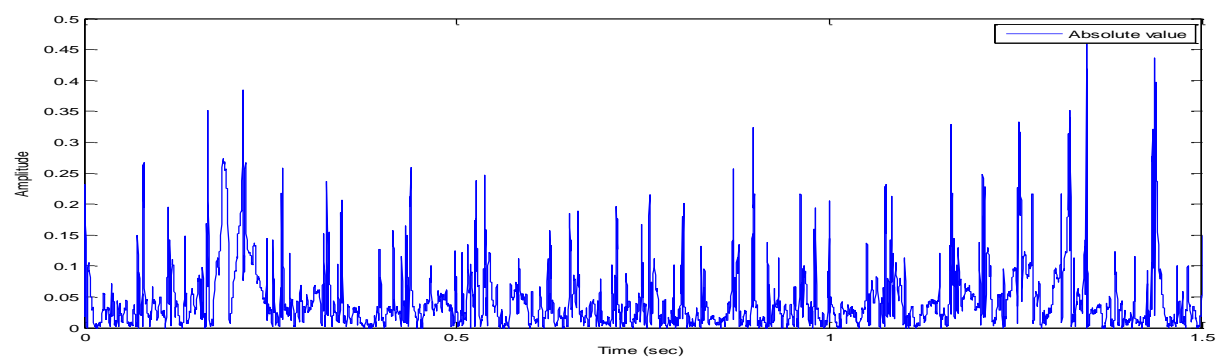

(c) 


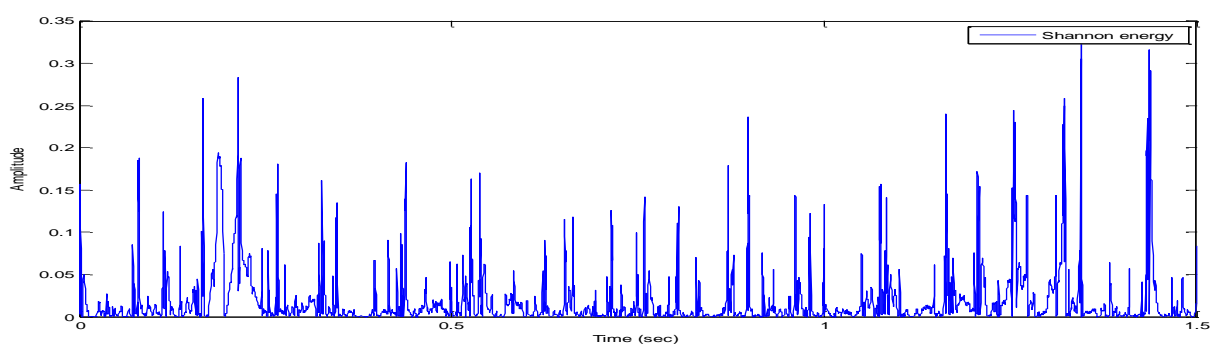

(d)

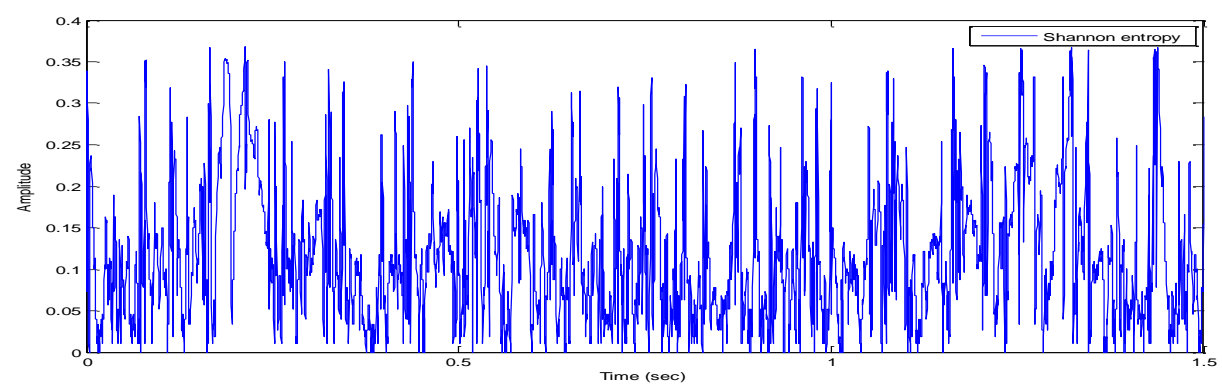

(e)

Figure 2: Energy representations of Normal EMG signal. (a) EMG signal. (b) Squared EMG signal. (c) Absolute value of the EMG signal. (d) Shannon energy of the EMG signal. (e) Shannon entropy of the EMG signal.

\section{Material and Methods}

\section{Database}

In this study, the database of EMG signal, includes three cases of EMGs signals (Normal, myopathy and Neuropathy), set from Medelec Synergy N2 EMG Monitoring System (Oxford Instruments Medical, Old Woking, United Kingdom).[6] A 25mm concentric needle electrode was placed into the tibialis anterior muscle (TA) of each subject. The patient was then asked to dorsiflex the foot gently against resistance. The needle electrode was repositioned until motor unit potentials with a rapid rise time were identified. Data were then collected for several seconds, at which point the patient was asked to relax and the needle removed. The following figure [Figure.3] and Table.I, shows three examples of EMG data from: a 44 year old man without history of neuromuscular disease; a 62 year old man with chronic low back pain and neuropathy due to a right L5 radiculopathy; and a $\mathbf{5 7}$ year old man with myopathy due to longstanding history of polymyositis, treated effectively with steroids and low-dose methotrexate. The data were recorded at $50 \mathrm{KHz}$ and then downsampled to $4 \mathrm{KHz}$. During the recording process two analog filters were used: a $20 \mathrm{~Hz}$ high-pass filter and a $5 \mathrm{~K} \mathrm{~Hz}$ low-pass filter. [Figure.3]

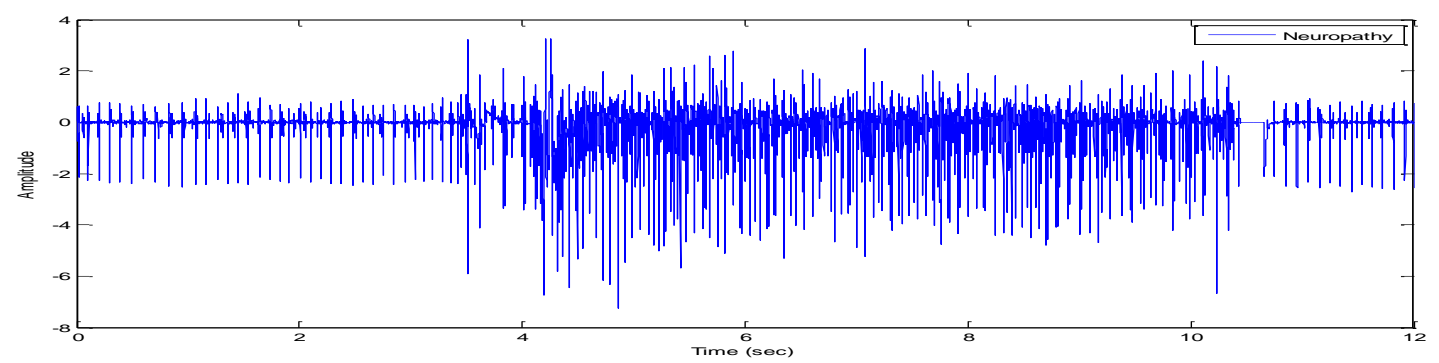

(c)

Figure 3: Temporal representation of Electromyography signals: (a) a normal case (b): a myopathy case (c): a neuropathy case.

\begin{tabular}{|c|c|c|c|c|c|}
\hline Signal & Age & Sex & Pathology & Muscle & Electrode \\
\hline EMG1 & 44 & Man & Any & tibialis anterior & A 25mm concentric needle electrode \\
\hline EMG2 & 57 & Man & Myopathy & tibialis anterior & A 25mm concentric needle electrode \\
\hline EMG3 & 62 & Man & Neuropathy & tibialis anterior & A 25mm concentric needle electrode \\
\hline
\end{tabular}

Table I: Database of EMGs signals. 


\section{Higher Order Statistics (HOS) theory for EMG signal analysis}

Frequency domain techniques are amongst the most fundamental and useful tools in the area of signal processing. Conventional techniques are generally based on the analysis of the first and second order moments and cumulants and their spectral representation. These techniques provide all the information available from the signal only if the underlying process is Gaussian and is operated on by a linear system.

For non-Gaussian processes and nonlinear systems, more information can be obtained from the higher order moments and cumulants (3rd order to Nth order) and their spectral representation (higher order spectra). The second order spectrum suppresses phase relationships, whereas information about the phase of the underlying system is available from higher order spectra [7].

Higher order statistics are useful in blind deconvolution and system recovery. As they do not suppress phase information, they may be applied to non-minimum phase and nonlinear systems. As they are able to recover information about non-Gaussian signals, they are useful in systems involving non-Gaussian input signals.

In this paper we will use, the Bispectrum, to estimate the system information. The bispectrum is the most accessible of the higher order spectra as it is the simplest to compute and its properties have been well explored [7] - [13].

\section{Non-linear parameters Analysis of normal and pathological EMGs signals:}

\section{a. Recurrence quantification analysis :}

Recurrence quantification analysis (RQA) is a nonlinear method that discovers recurring patterns, commonly referred to ashidden rhythms. This nonlinear method can be used to analyze physiological signals. To be specific, recurrence plots are used to investigate the nonlinear prediction of timeseries generated by dynamical systems driven by slowly varying external forces. Recurrence plots are established based on time series data. The first step is to form a vector:

$$
X_{n}=[x(n) x(n+L), \ldots, x(n+(m-1) L)]
$$

Where $\mathbf{m}$ is the embedding dimension and Lis the lag. $\mathbf{X n}$ represents a multi-dimensional process as a trajectory in m-dimensional space. We can define two different points on that trajectory, say $\mathbf{X i}$ and $\mathbf{X j}$. The index pair $(i, j)$ is used to specify an element for a binary $N^{*} N$ matrix. The entry is defined as

$$
a_{i, j}=\left\{\begin{array}{ccc}
1 & \text { if } & \left\|X_{i}-X_{j}\right\| \leq r \\
0 & & \text { otherwise }
\end{array}\right\}
$$

Where $\|. .$.$\| indicates the Euclidean distance andris a fixed radius. The$ binary matrix $\mathbf{A}$ can be represented as a $\mathrm{N}^{*} \mathrm{~N}$ image by putting a white pixel whenever $a_{i, j}=0$ and a blue pixel otherwise.

The recurrence statistics, which is called recurrence quantification analysis (RQA), has been introduced for measuring quantitative information within recurrence plots [14]; subsequently it was extended with new measure of complexity by Marwan [15] [16]. It is a method of nonlinear data analysis which quantifies the numbers and duration of recurrence of a dynamical system presented by its state space vector. An EMG recurrence plot, which includes the recurrence statistics, is a representation of the nonlinear EMG signal that provides rich information about EMG patterns. We focus on the following three features of the recurrence plot as they best describe the behaviour of the underlying EMG signals:

a. Recurrence rate (RR) is the density of recurrence points in a recurrence plot, which is defined as:

$$
R R=\frac{1}{N^{2}} \sum_{i, j}^{N} R_{i, j}
$$

Where $\mathrm{N}$ is number of points on the phase space trajectory

b. The next measure is the percentage of recurrence (determinism: DET) points that form diagonal structures to all recurrence points in the recurrence plot $(\mathrm{R})$ reflecting deterministic or predictable characteristics in dynamical systems [17] The line segments are parallel to the main diagonal. Higher value of DET means higher periodicity. The vector point ( $i$ and $j$ ) in the recurrence plot is considered as recurrent if the distance between the vectors) $y_{i}$ and $y_{j}$ is less than the threshold. The value of DET is given as below. The DET is usually expressed as a percentage. In this analysis, the DET value is taken between 0 and 1 for representation.

$$
D E T=\frac{\sum_{l=l_{\min }}^{N} l P(l)}{\sum_{l=1}^{N} l P(l)}
$$

Where $P(l)$ is the frequency distribution of the lengths lof the diagonal lines.

c. Complexity feature (CPX) is derived from the RQA measure determinism. This feature is previously reported for analyzing physiological signals (EEG) and found to be useful [18] Higher value of CPX indicates origin of signal from highly irregular or chaotic system. This may be due to presence of random series and may be due to higher nonlinearity. Its represented as :

$$
C P X=-20 \log _{10} D E T
$$

(9)

\section{b. Bispectral Analysis}

Bispectrum analysis reveals the phase relation between components of a signal [8][19]. Unlike the power spectrum, the bispectrum is capable of extracting extra information from biological signals such as an EMG signal, which is non-Gaussian and nonlinear. The bispectrum is defined as the Fourier transform of the 3rd-order cumulant.

The 3rd-order cumulant of a discrete signal $x(k)$, which is stationary and has a 0 mean, is defined as [19]

$\boldsymbol{C}_{3 x}\left(\boldsymbol{n}_{1}, \boldsymbol{n}_{2}\right)=\operatorname{cum}\left\{x(k) x\left(k+n_{1}\right) x\left(k+n_{2}\right)\right\}=\langle x(k) x(k+$ $\left.\left.n_{1}\right) x\left(k+n_{2}\right)\right\rangle-\langle x(k)\rangle\left\{\left\langle x(k) x\left(k+n_{1}\right)\right\rangle+\left\langle x(k) x\left(k+n_{2}\right)\right\rangle+\right.$ $\left.\left\langle x\left(k+n_{1}\right) x\left(k+n_{2}\right)\right\rangle\right\}+2\langle x(k)\rangle^{3}$

Where $\langle\cdot\rangle$ denotes the expected process.

The $r$ th degree moment of $x(k)$ is defined as

$m_{r x}\left(n_{1}, n_{2}, \ldots, n_{r-1}\right)=\left\langle x(k) x\left(k+n_{1}\right) \cdots x\left(k+n_{r-1}\right)\right\rangle$ 
Thus, (10) can be rewritten as:

$C_{3 x}\left(n_{1}, n_{2}\right)=m_{3 x}\left(n_{1}, n_{2}\right)-\left(m_{x}\left(m_{2 x}\left(n_{1}\right)+m_{2 x}\left(n_{2}\right)+m_{2 x}\left(n_{2}-n_{1}\right)\right)-\right.$ $2 m_{3 x}$ )

Alternatively, the 3rd-order cumulant can be written as

$C_{3 x}\left(n_{1}, n_{2}\right)=m_{3 x}\left(n_{1}, n_{2}\right)-m_{3 x}^{G}\left(n_{1}, n_{2}\right)$

where $m_{3 x}\left(n_{1}, n_{2}\right)$ is the 3rd-order moment function of $x(k)$ and $m_{3 x}^{G}\left(n_{1}, n_{2}\right)$ is the 3 rd-order moment function of a Gaussian random process with the same 1 st- and 2nd-order characteristics of $x(k)$

$m_{3 x}^{G}\left(n_{1}, n_{2}\right)=m_{x}\left(m_{2 x}\left(n_{1}\right)+m_{2 x}\left(n_{2}\right)+m_{2 x}\left(n_{2}-n_{1}\right)\right)-2 m_{3 x}$

The Fourier transform of the 3rd-order cumulant is bispectrum and defined as

$\mathrm{B}\left(w_{1}, w_{2}\right)=\sum_{\mathrm{n} 1=-\infty}^{+\infty}=\sum_{\mathrm{n} 2=-\infty}^{+\infty}=\boldsymbol{C}_{3 x}\left(n_{1}, n_{2}\right) W e^{-j(w 1 n 1+w 2 n 2)}$, $|w 1|,|w 2| \leq \pi$

where $W\left(n_{1}, n_{2}\right)$ is the 2-dimensional window function that decreases the variance of the bispectrum. In this study, a Hanning window was used. Equation (15) can also be defined in the Fourier transform of $x(k)$ as

$\mathrm{B}\left(w_{1}, w_{2}\right)=\left\langle X\left(w_{1}\right) X\left(w_{2}\right) X^{*}\left(w_{1}+w_{2}\right)\right\rangle$
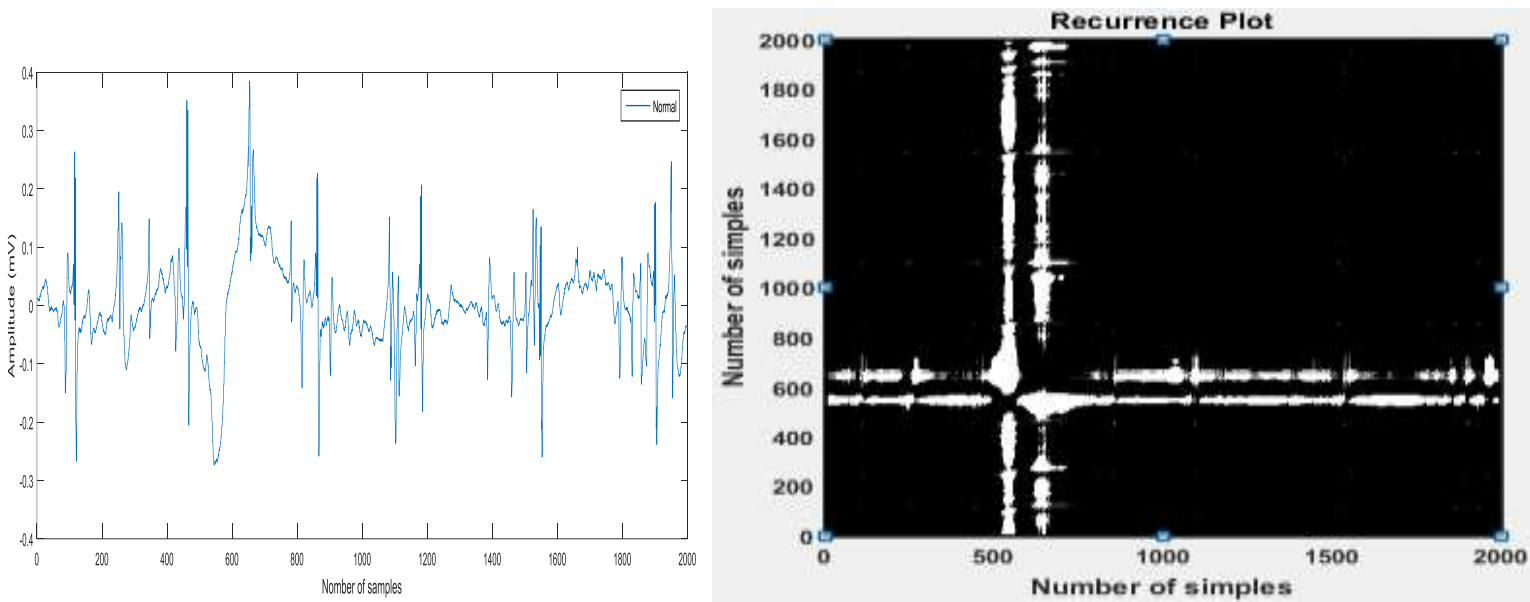

(a) where $*$ denotes a complex conjugate.

$B\left(\omega_{1}, \omega_{2}\right)$ is a symmetric function, such that a triangular region $\mathbf{0} \leq \omega_{2} \leq$ $\omega_{1}, \omega_{1}+\omega_{2} \leq \pi$ could completely describe the whole bispectrum. The other regions in the bispectrum are the symmetry of the defined triangular region.

\section{Results and discussion}

Analyzing nonlinear time series through recurrence quantification analysis has been investigated for many years [20] [21]. RQA has been proven to be effective in analyzing nonstationary signals. Not unexpectedly, RQA has demonstrated advantages over linear techniques in the analysis of EMG [22]. To test whether electromyogram is a nonlinear signal or just random noise, we analyze EMG signals of neuromuscular diseases using recurrence plot [see Figure. 4].

The recurrence plots, shown in Figure.4, describe the natural, but subtle, time correlation within the EMG signals. The recurrence plots are almost symmetric with respect to the diagonal $\mathrm{i}=\mathrm{j}$.

The recurrence plots for normal case and Myopathy case are quite distinct compared to Neuropathy case. This is because a recurrence plot picks up similarities in the EMG signals, therefore, the recurrence plot reflects the degree of similarity present in the EMG signal.

From all the results, we conclude that EMG obeys a certain nonlinear deterministic law and non-stationarity is significant within these signals. It is therefore possible to study and analyze these signals as a non-linear system using recurrence quantification analysis. This result confirms what is found by Sivarit Sultornsanee, Ibrahim Zeid and Sagar Kamarthi [23] 


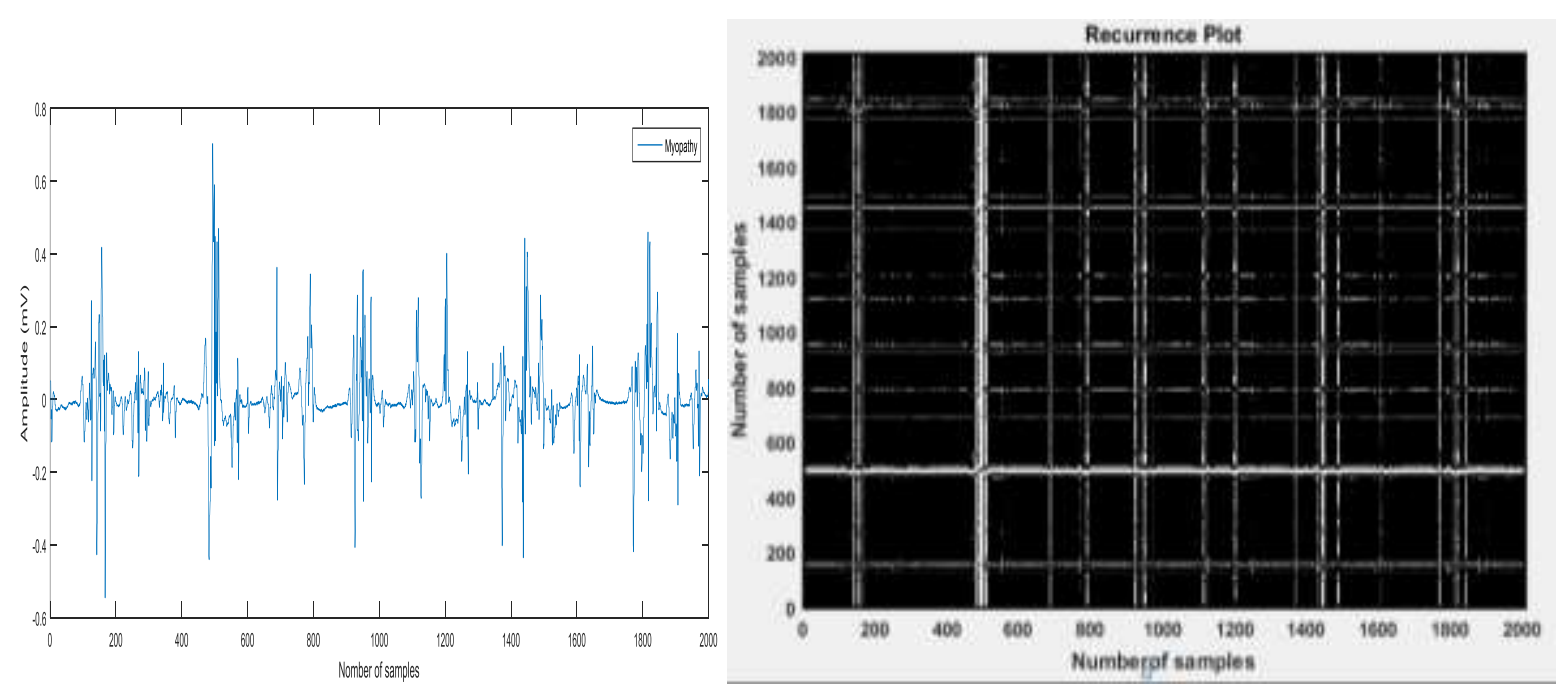

(b)
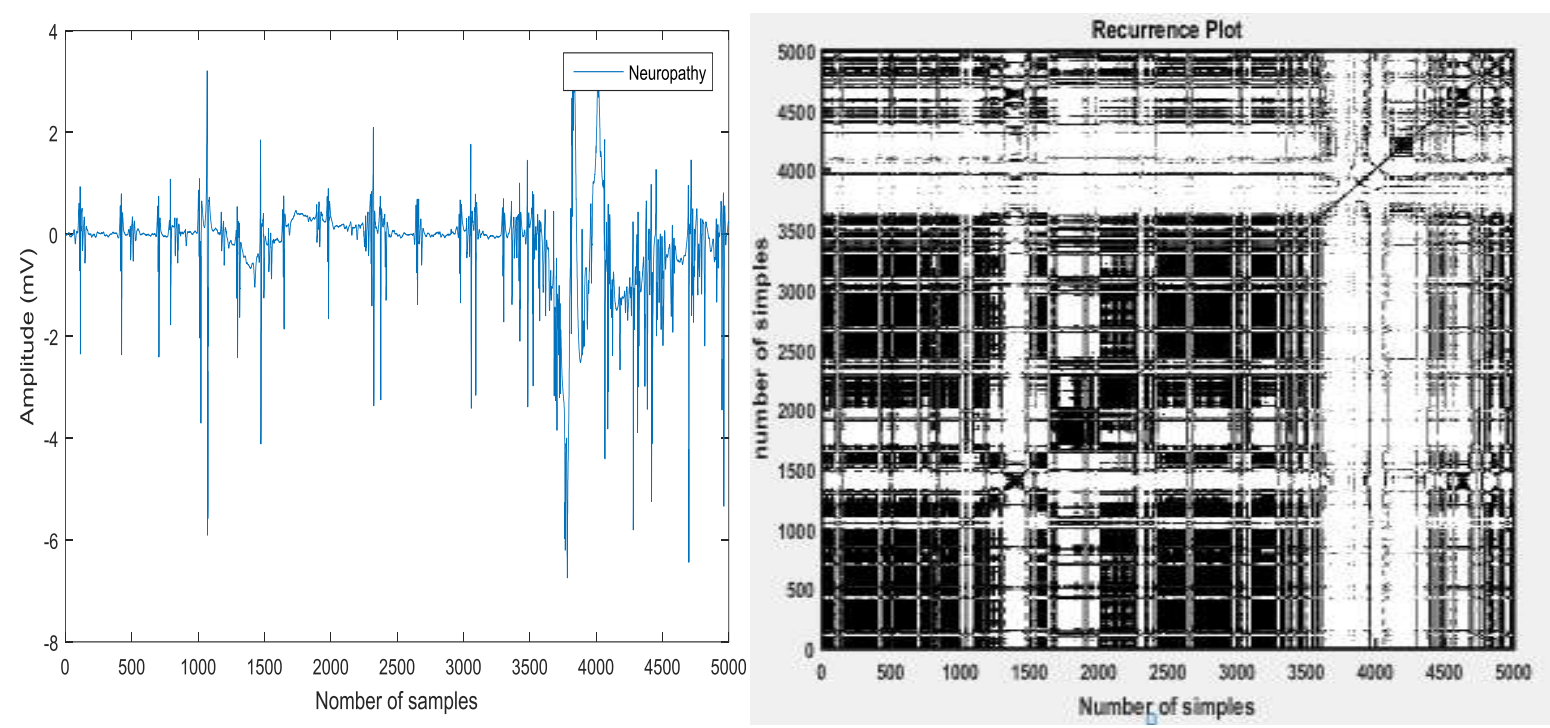

(c)

Figure 4: Recurrence plots of Electromyography signals: (a) a normal case (b): a myopathy case (c): a neuropathy case.

To distinguish the difference between the EMGs signals and hence to estimate the evolution of their disease severity, histograms of the variation of the same parameters mentioned previously given in the table below [Table. II] (Recurrence rate (RR), Determinism (DET\%) and Complexity feature (CPF)) will be plotted. Thus, a comparison of these cases will be performed. [Figure.5]

The histogram of the Recurrence rate (RR) variation represented by [Figure.5. (a)], shown an inversely relative proportionality between the value of the Recurrence rate (RR) and the type of the signal. The first recording (the normal case) represents a higher value $(\approx 181)$, that the other case. The second with a myopathy, also has an important value, closer to the normal case (165.5) and more important than the neuropathy case $(\approx 47.7)$. This variation shows that the myopathy case has properties similar to the normal case that neuropathy case.

The DET feature is the measure of determinism and periodicity in time series. The lower DET is an indication of higher randomness and lower predictability.[24]

For the same cases studied, the result of the variation in DET shown in Figure.5. (b), makes clear the differences that may exist between these signals. For the normal case, the EMG signal is highly random and nonstationary in nature. This may be due to higher firing rates of motor units and increased recruitment of motor units for force generation. However, for the pathological cases, the DET value appeared to be increase steadily to higher values. 
Here, the DET value of myopathy, are much closer to the normal case as the neuropathy. These results, so well confirm what was found by the recurrence rate (RR) and consequently the myopathy is less severe than the neuropathy. These findings are confirmed by those given in [23].

Complexity feature variations of three different cases studied represented by [Figure.5. (c)], shows that the first recording (the normal case) is the least case. It represents $16 \%$ of the value of myopathy and about $5 \%$ of the neuropathy case. Higher complexity means that the signal is random and highly non stationary. Lower values of complexity are an indication of repetitive regular structures and hence considered to be predictable. [24]. In this context the CPX variation, present an important parameter to complete differentiation and classification pathological severity between EMGs signals: Neuropathy is the most severe cases.
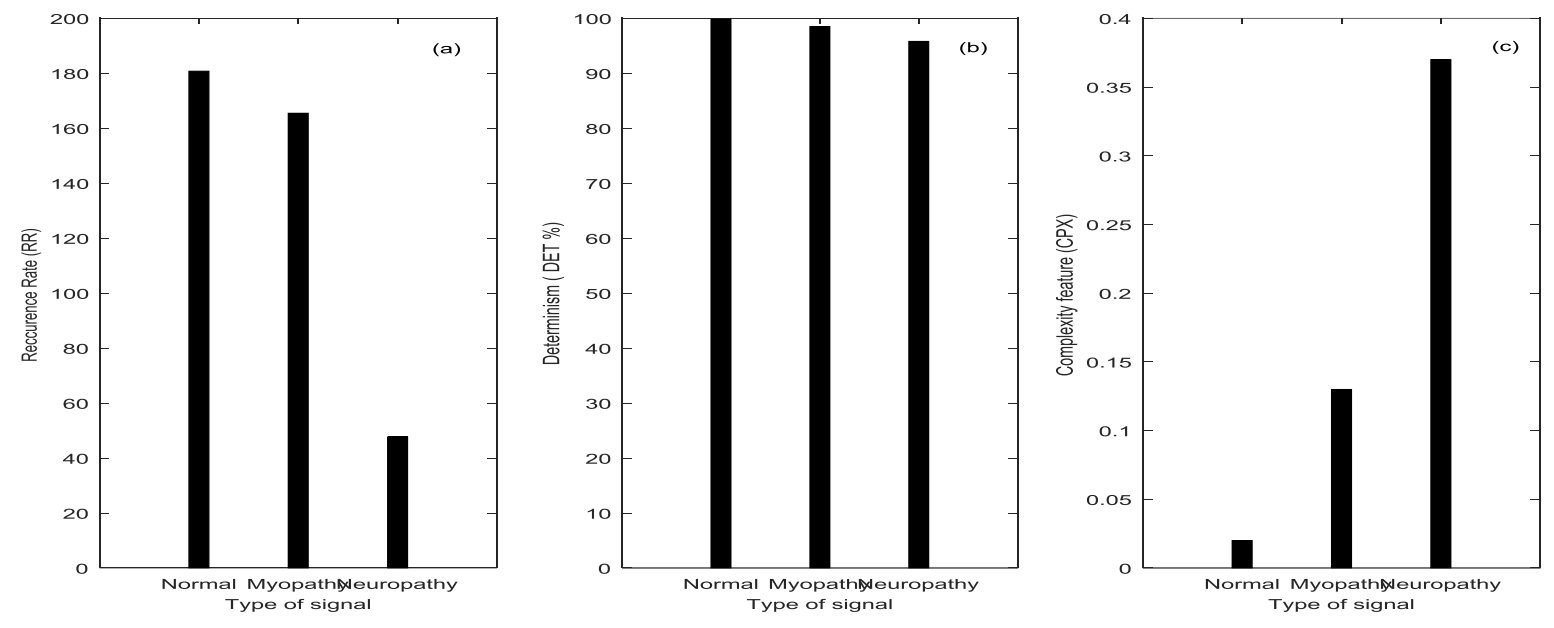

Figure 5: histograms of the variation of the non-linear parameters

\begin{tabular}{|l|l|l|l|}
\hline Signal & RR & DET (\%) & CPX \\
\hline Normal & 166.4477 & 99.68 & 0.02 \\
\hline Myopathy & 165.5 & 98.52 & 0.13 \\
\hline Neuropathy & 47.6976 & 95.8296 & 0.37 \\
\hline
\end{tabular}

Table II: Nonlinear parameters analysis of the EMGs signal

The frequency representation by applying the bispectrum analysis [Figure.6, Figure.8 and Figure.10 ] and the estimating study of the frequency and amplitude variation given by the diagonal slice : $A m p=f(F r e q)$ of the three cases of EMGs signals [ Figure.7, Figure.9 and Figure.11]: normal, myopathy and neuropathy, shows that important frequencies to maintain the muscle signal range from 20 to 500 $\mathrm{Hz}$.

For the first case, the resulting bispectrum [Figure.6. (b)], shows a well contoured representation with maximum amplitude for a frequency of
$20 \mathrm{~Hz}$. Thus, the most important energy is conserved in the range 20 to $120 \mathrm{~Hz}$. The diagonal slice of a portion of the bispectral representation proves what was represented before and shows that the maximum amplitude is $0.41 \mathrm{mV}$ for a frequency of $20 \mathrm{~Hz}$, from amplitude $0.08 \mathrm{mV}$ and frequency $50 \mathrm{~Hz}$ the information begins to decrease until $95 \mathrm{~Hz}$, beyond that the information begins to disappear. [Figure.7]

The second case with a electromyopathy signal, the resulting bispectrum analysis shows a primary deformation of the contour, as well as a dilation towards high frequencies with maximum amplitude for a frequency of 32 Hz. Thus, the most important energy is conserved in the range 20 to 200 Hz. [Figure.8. (b)] 


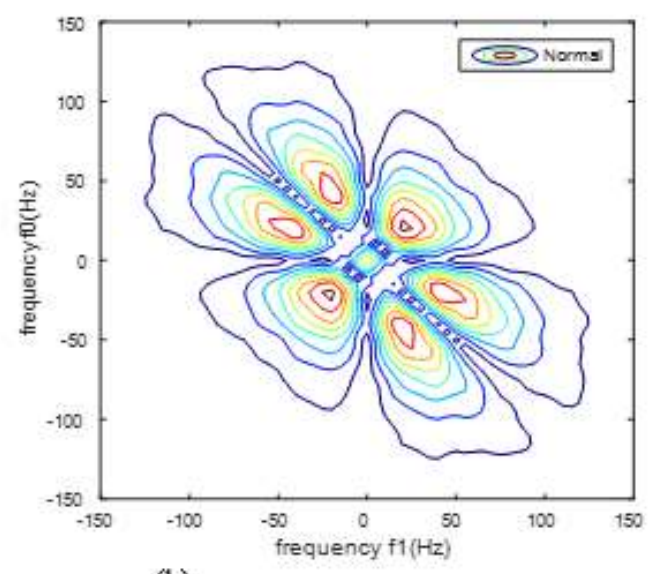

(b)

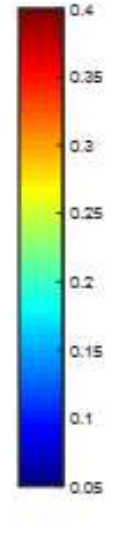

(c)

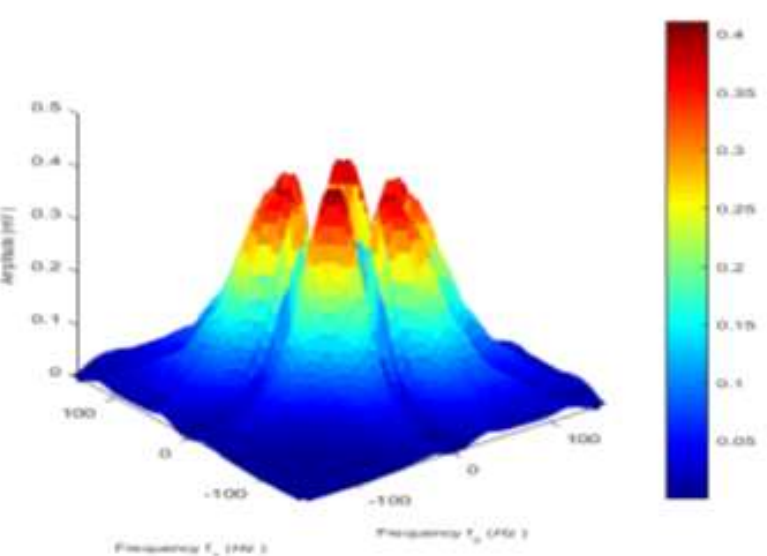

Figure 6: (a): Temporal representation of a normal case, (b) and (c): bispectral representation (contour in (a), surface in (b))

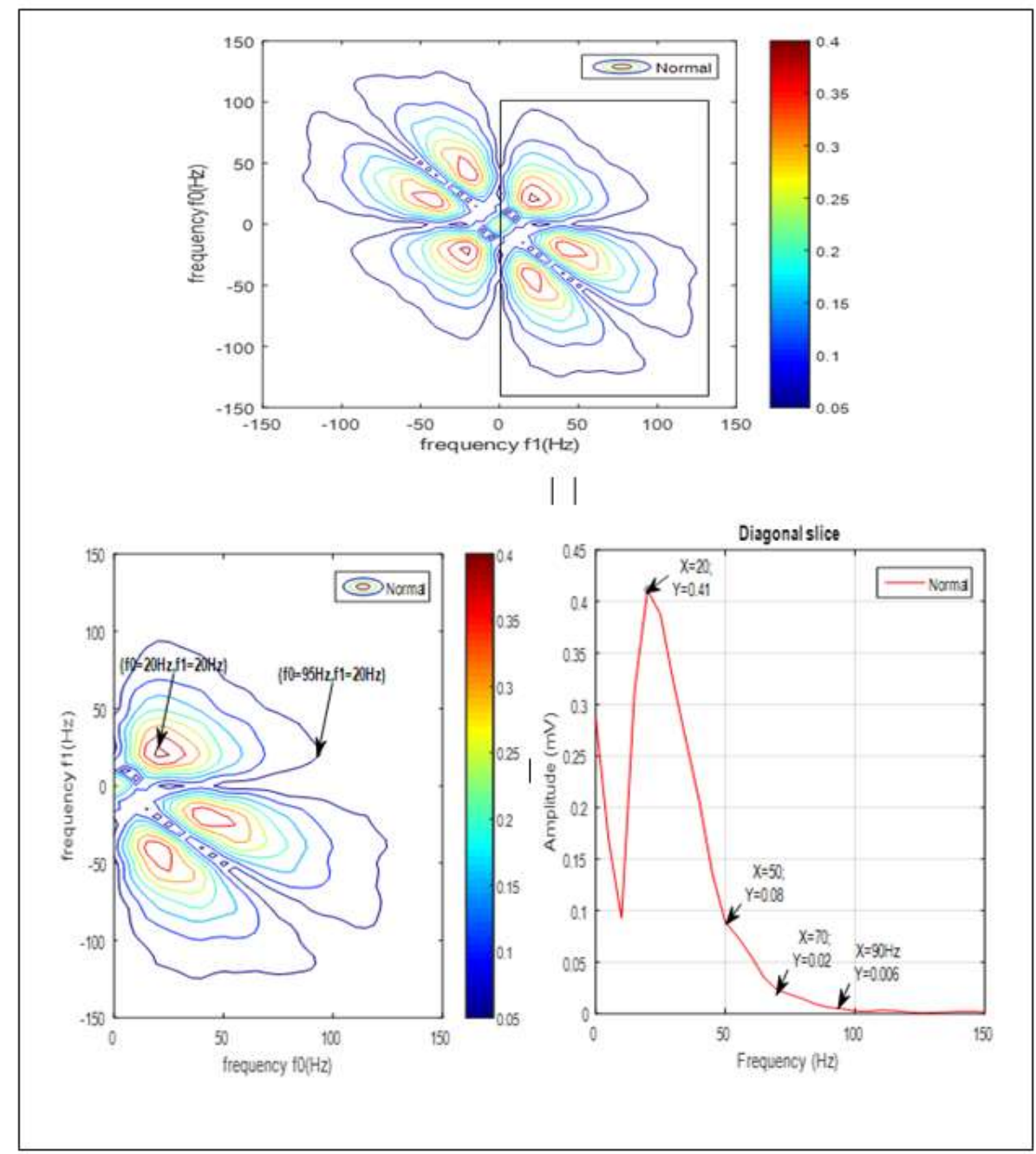

Figure 7: The diagonal slice of a portion of the bispectral representation of a normal case. 


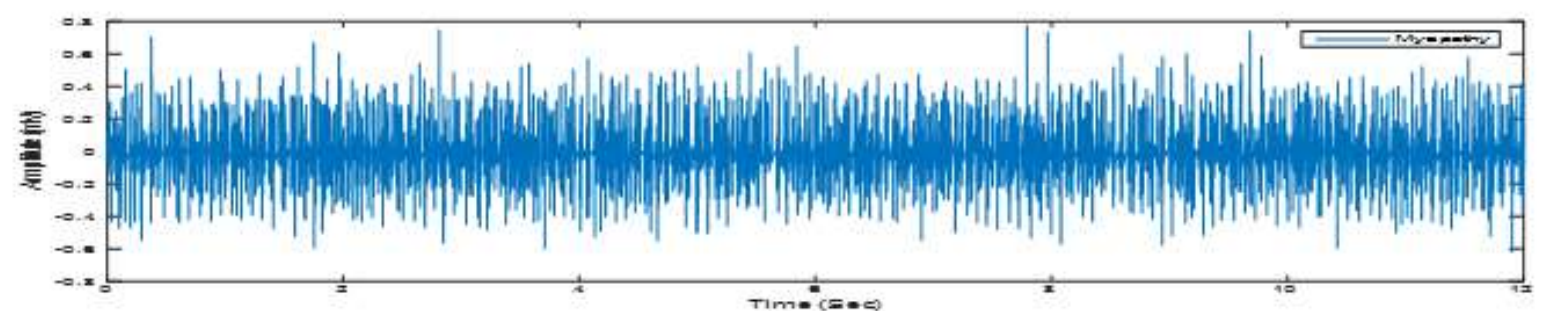

(a)

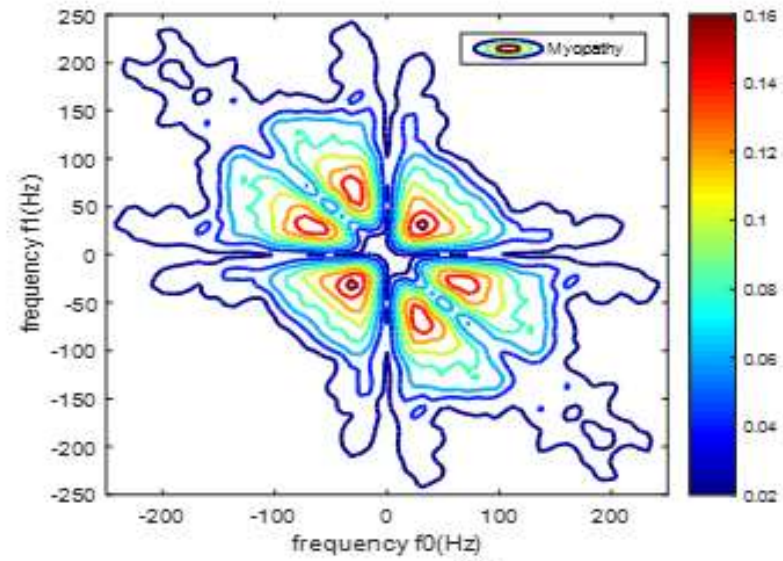

(b)

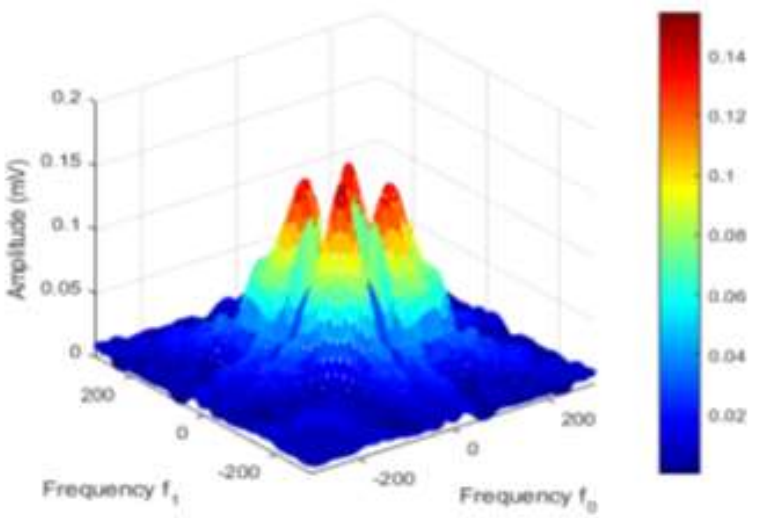

(c)

Figure 8: (a): Temporal representation of a Myopathy case, (b) and (c): bispectral representation (contour in (a) and surface in (b))

The diagonal slice of a portion of the bispectral representation shows also that the maximum amplitude is $0.16 \mathrm{mV}$ for a frequency of $32 \mathrm{~Hz}$, from amplitude $0.01 \mathrm{mV}$ and frequency $88 \mathrm{~Hz}$ the information begins to decrease to $216 \mathrm{~Hz}$, beyond that the information begins to disappear. [Figure.9]

The third case for a neuropathic type, the application of the bispectrum analysis and the diagonal slice of a portion of the bispectral representation, proves again that the most suitable frequency range to analyze EMG signals is 20 to $500 \mathrm{~Hz}$. In this pathological type, the signal bispectrum is a contour very distorted and dilated towards the high frequencies with maximum amplitude for the frequency of $60 \mathrm{~Hz}$. Thus, energy is conserved in the range 20 to $480 \mathrm{~Hz}$. [Figure.10. (b)]

The diagonal slice of a portion of the bispectral representation shows that the maximum amplitude is $9.9 \mathrm{mV}$ for a frequency of $60 \mathrm{~Hz}$, from amplitude $0.84 \mathrm{mV}$ and frequency $270 \mathrm{~Hz}$ the information starts to decrease until $400 \mathrm{~Hz}$ at $500 \mathrm{~Hz}$, beyond the information begins to disappear. [Figure.11]

The time domain representation (higher-order cumulants) [Figure.12] of the three cases of EMGs signals: normal, myopathy and neuropathy, shows that: for the first case, the resulting cumulant shows a well contoured representation with maximum information is located in the center. Thus, the most important energy is conserved in the very short range (few ms.). [Figure.12. (a)]. The second case with a electromyopathy signal, the resulting cumulant representation shows a primary deformation of the contour, with a large dilation, with a maximum amplitude going up to $5^{*} 10^{-4} \mathrm{mv}$. Thus, the most important energy is conserved around to $5^{*} 10^{-3} \mathrm{~ms}$. [Figure.12. (b)]. The third case for a neuropathic type, the application of the cumulant analysis show a contour very distorted and dilated towards $0.05 \mathrm{~ms}$. with maximum amplitude $0.1 \mathrm{mv}$. [Figure.12. (c)] 


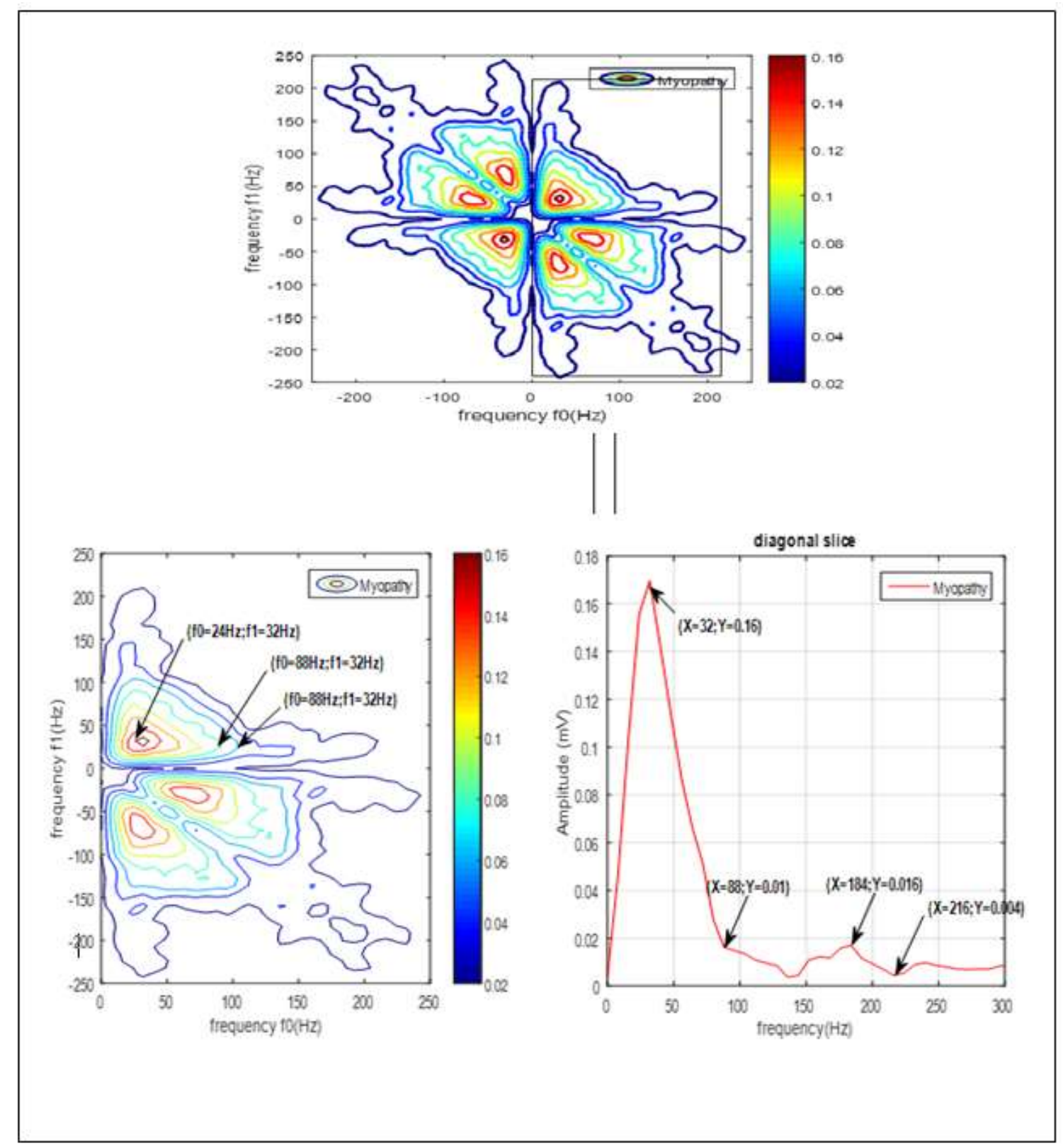

Figure 9: The diagonal slice of a portion of the bispectral representation of a Myopathy case. 


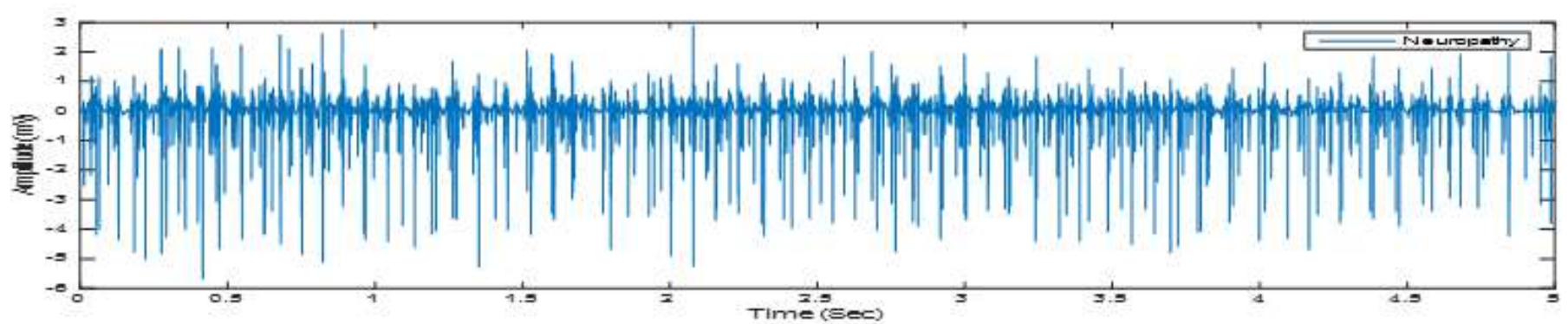

(a)

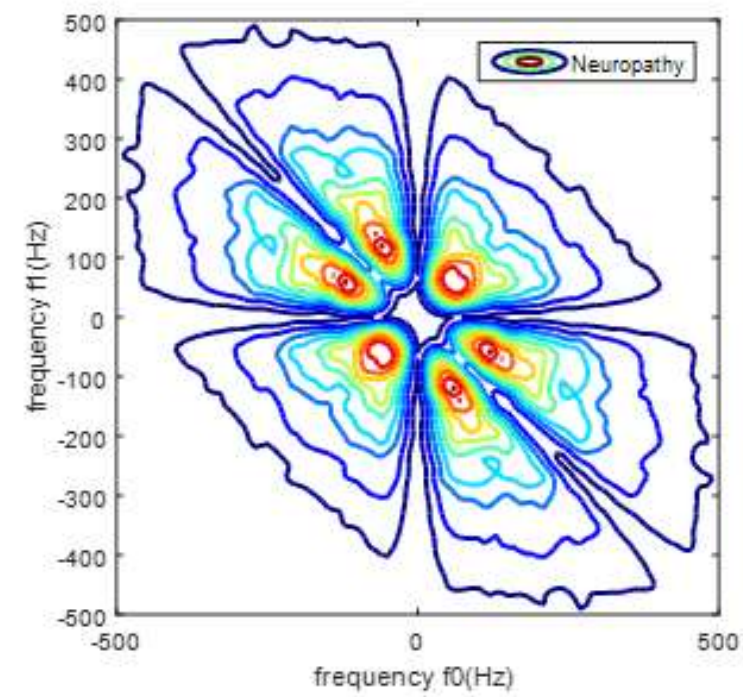

(b)

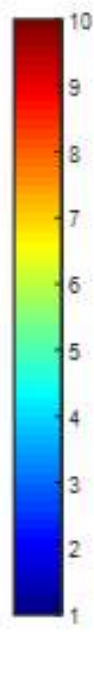

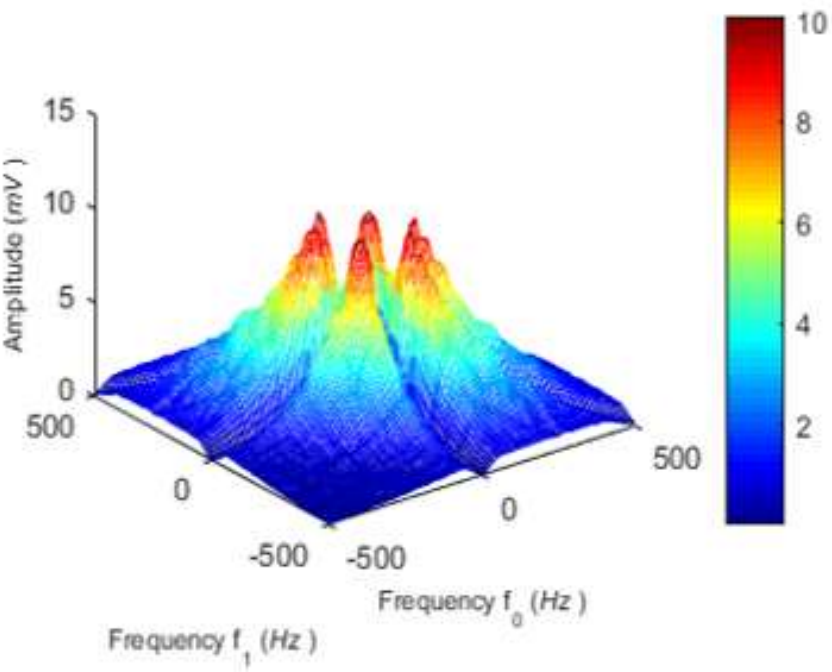

(c)

Figure 10: (a): Temporal representation of a Neuropathy case, (b) and (c): bispectral representation (contour in (a) and surface in (b) 


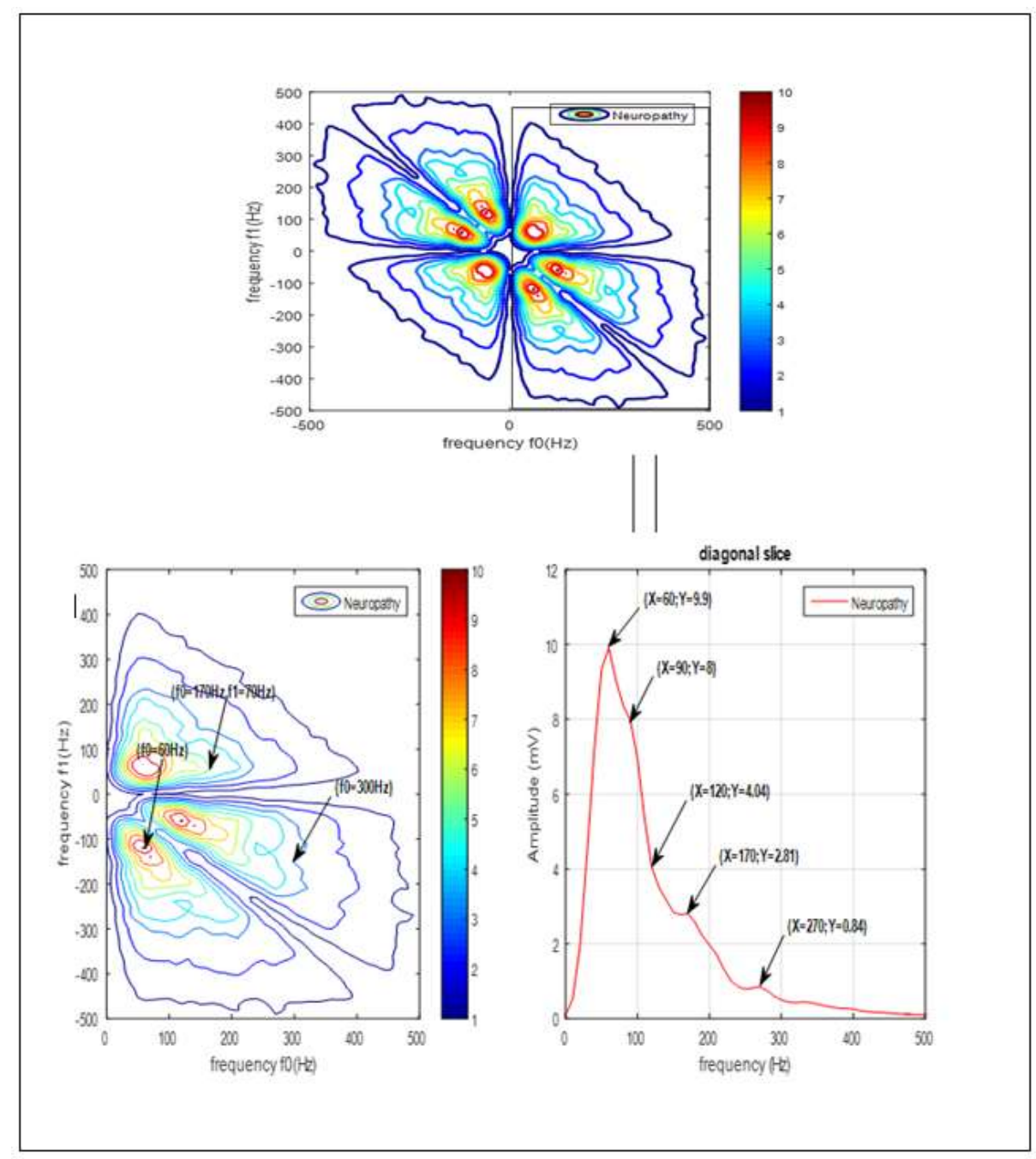

Figure 11: The diagonal slice of a portion of the bispectral representation of a Neuropathy case.

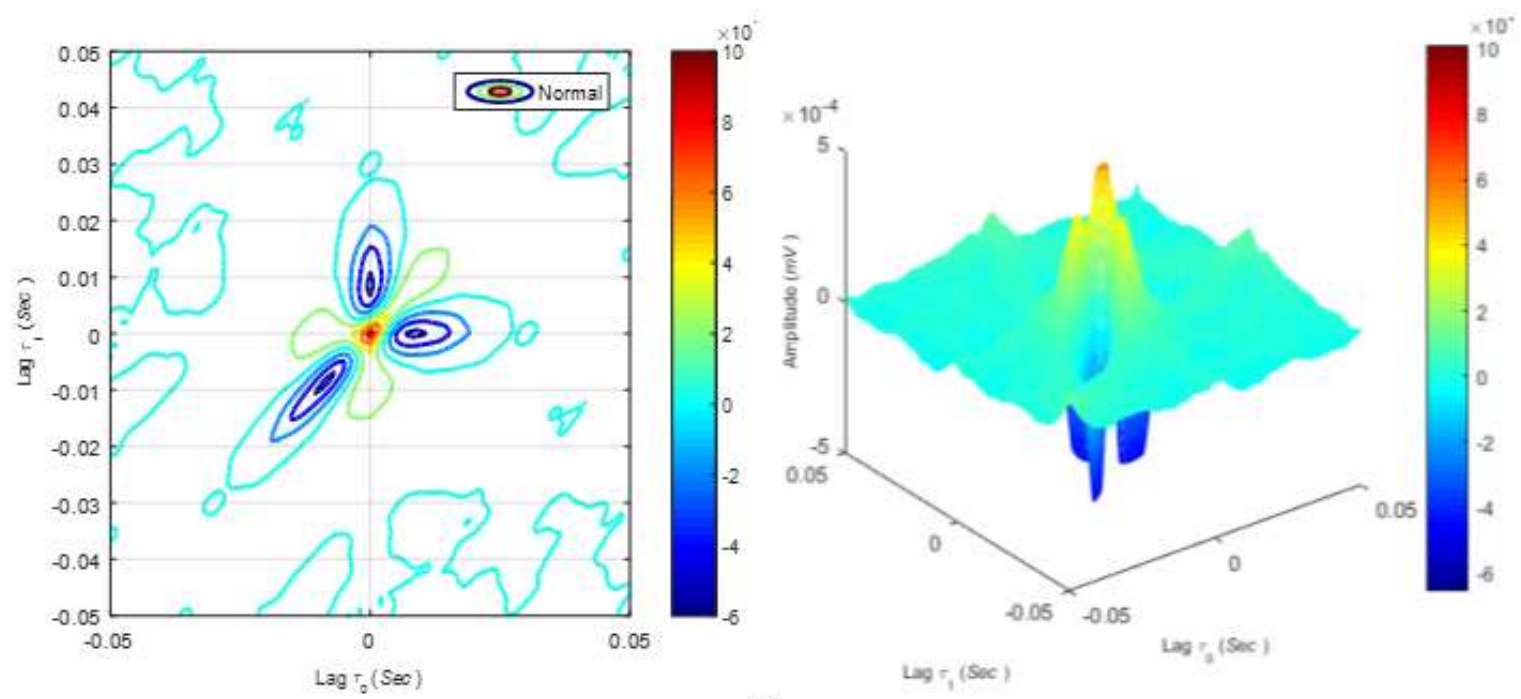

(a) 

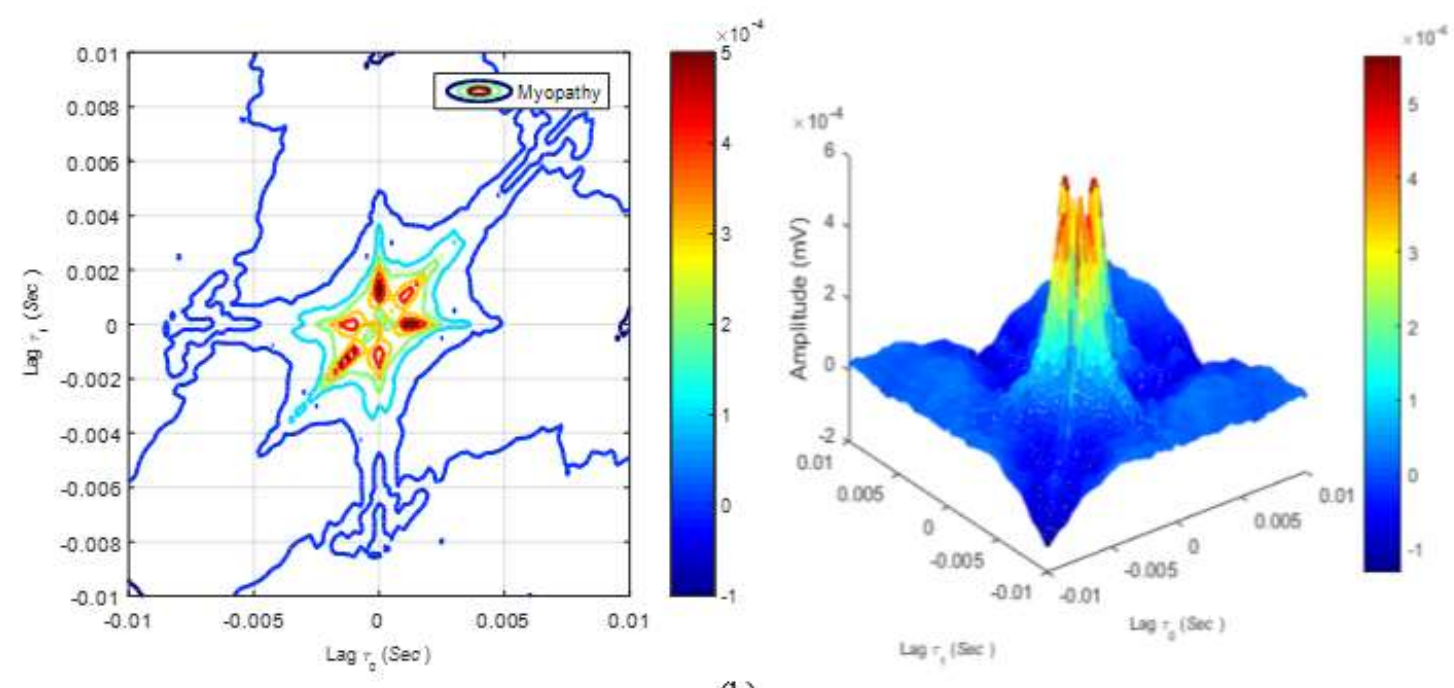

(b)
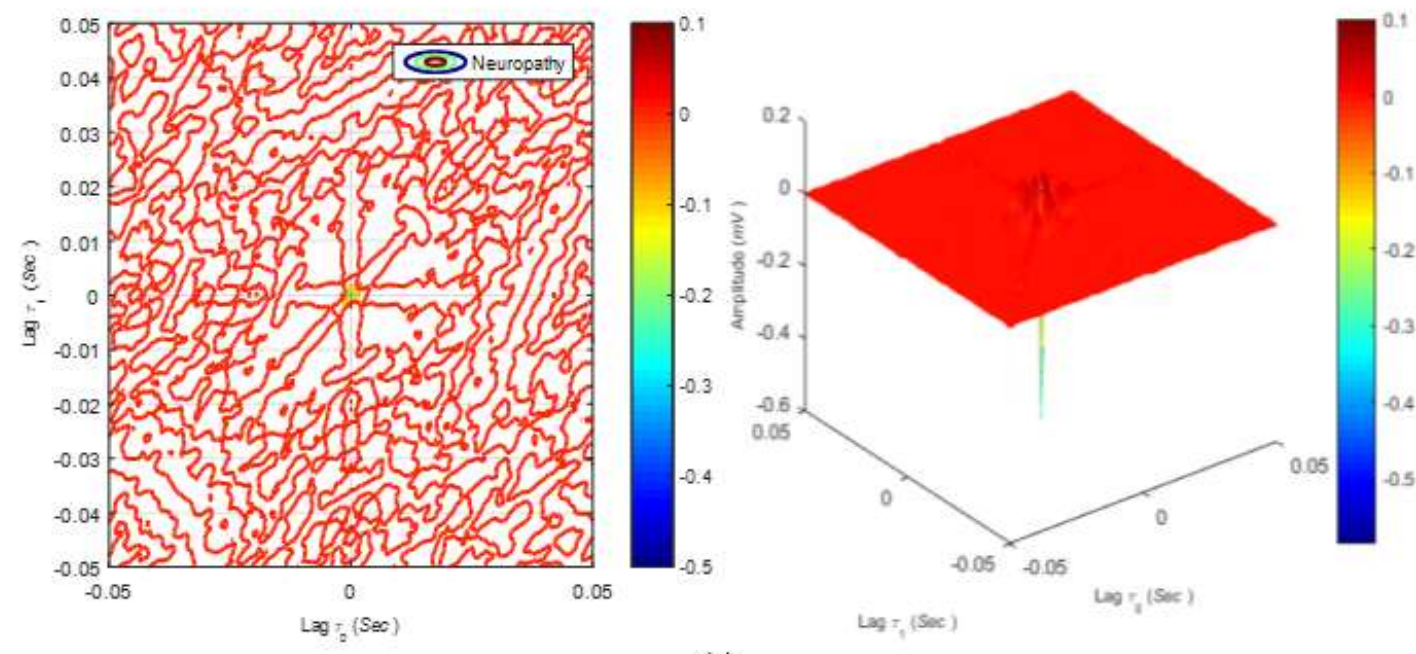

(c)

Figure 12: Cumulant plots (contour and surface) for: (a) Normal case, (b): Myopathy case and Neuropathy case in (c).

\section{Conclusion:}

Electromyography plays an important role in clinical neurological diagnosis; it can confirm or dismiss clinical diagnoses, indicate the location and type of an abnormality or expose disorders that are clinically uncertain.

Several methods based on modern signal Processing techniques, have been investigated.

The non lineaire analysis of the EMG signal based on the recurrence quantification analysis (RQA) and bispectrum representation, was shown to be particularly promising for the detection of muscle status changes. It appears that RQA and bispectrum methods may be a useful technique in differentiating pathological cases from normal cases. The results obtained from this analysis are acceptably high enough. This simple and effective method may help experts in defining pathological cases and this can give important clues about some abnormalities related to EMG signals.

\section{Ethical statement}

- $\quad$ No, I have nothing to report

- This study was not funded by any party: it is an academic $\mathrm{PhD}$ study
- No conflict of interest

- No animal or other used in this study

- all authors were involved in the work leading up to the manuscript.

- The results are appropriately placed in the context of prior and existing research.

- All sources used are properly disclosed (correct citation).

Acknowledgements

The authors would like to thank the Directorate-General of Scientific

Research and Technological Development (Direction Générale de la Recherche Scientifique et du Développement Technologique, DGRSDT, URL: $w w w . d g r s d t . d z$, Algeria) for the financial assistance through a monthly allowance provided to the doctoral students to do their research. 


\section{Reference:}

1. D. Farina , L. Fattorini, F. Felici, G. Filligoi, "Nonlinear surface EMG analysis to detect changes of unit conduction velocity and synchronization", J Appl Physiol, vol 93,no 0.5 ,pp. 1753-1763, 2002.

2. D. Rangaprakash, "Connectivity analysis of multichannel EEG signals using recurrence-based phase synchronization technique,” Comput. Biol. Med., vol. 46, pp. 11-21, Mar. 2014.

3. K. Ito and Y. Hotta, "EMG-based detection of muscle fatigue during low-level isometric contraction by recurrence quantification analysis and monopolar configuration," in Proc. Conf. Proc. IEEE Eng. Med. Biol. Soc., vol. 2012, no. 257, pp. 4237-4241, Jan. 2012.

4. C. L. Webber and J. P. Zbilut, "Dynamical assessment of physiological systems and states using recurrence plot strategies.," J. Appl. Physiol., vol. 76, no. 2, pp. 965-73, Feb. 1994.

5. N. Sezgin, "Analysis of EMG Signals in Aggressive and Normal Activities by Using Higher-Order Spectra", The Scientific World Journal, vol. 2012, Article ID 478952, 5 pages, 2012.

6. D. Stachuk, T.A. 'Clinical quantitative electromyography", INTECH, Mai,2013.

7. C. L. Nikias and M. R. Raghuveer, "Bispectrum estimation: A digital signal processing framework," Proceedings of the IEEE, vol. 75, no. 7, pp. 869- 891, 1987.

8. C. L. Nikias and. A. P. Petropulu, Higher-Order Spectral Analysis: A Nonlinear Signal Processing Framework. Englewood Cliffs, NJ: PTR Prentice Hall, 1993.

9. S. Shahid and J. Walker, "The complex cepstrum of bispectrum for system reconstruction with application to sEMG signal," in Proceeding of the Irish Signals and Systems Conference, pp. 230-235, 2003.

10. S. Shahid, Higher Order Statistics Techniques Applied to EMG Signal Analysis and Characterization. Ph.D. thesis, University of Limerick, Ireland, 2004.

11. T. S. Rao and M. M. Gabr, "A test for linearity of stationary time series," vol. 1, no. 1, pp. 145-158, 1980.

12. M. Rangoussi and G. B. Giannakis, "FIR modeling using logbispectra: Weighted least-squares algorithms and performance analysis," IEEE Transactions on Circuits and Systems, vol. 38, no. 3, pp. 281-296, 1991.

13. J. C. Marron, P. P. Sanchez and R. C. Sullivan, "Unwrapping algorithm for least-squares recovery from the modulo $2^{1 / 4}$ bispectrum phase," J. Opt. Soc. Am. A, vol. 7, no. 1, pp. 14-20, Jan. 1990.

14. J.P. Zbilut, M. Koebbe, H. Loeb, and G. Mayer-Kress, ' use of recurrence plots in the analysis of heart beat intervals, Proceedings of the IEEE Conference on Computers in Cardiology, pp. 263-266, 1992.

15. N. Marwan, N. Wessel, U. Meyerfeldt, A. Schirdewan, and J. Kurths, "recurrence plot-based measures of complexity and application to heart rate variability data", Physical Review E, vol.66, no.2, 2002.

16. J.P. Eckmann, S.O. Kamphorst, D. Ruelle, and S. Ciliberto, "liapunov exponents from time series", Physical Review A, vol.34, no.6, pp. 4971-4979, 1987.

17. K. Li and Z. M. Li, "Cross recurrence quantification analysis of precision grip following peripheral median nerve block," J. Neuroeng. Rehabil., vol. 10, p. 28, Jan. 2013.

18. K. Becker, G. Schneider, M. Eder, A. Ranft, E. F. Kochs, W. Zieglgänsberger, and H. U. Dodt, "Anaesthesia monitoring by recurrence quantification analysis of EEG data," PLoS One, vol. 5, no. 1, p. e8876, Jan. 2010

19. J.C.Sigl and, N.G. Chamoun "An introduction to bispectral analysis for the electroencephalogram", Journal of Clinical Monitoring, Vol. 10, No. 6, pp.392-404.1994

20. N. Marwan, M.C. Romano, M. Thiel, and J. Kurths, "recurrence plot for the analysis complex systems", Physics Reports, pp. 237-329, 2007.

21. G. Filigoi, F. Felici, N. Vicini, and A. Rosponi, ,'recurrence quantification analysis of surface electromyogram", Proceedings of the Mediterranean Conference on Medical and Biological Engineering and Computing, 1998.

22. Liu, Y., Kankaanpää, M., Zbilut, J. et al. EMG 'recurrence quantifications in dynamic exercise'. Biol. Cybern. Vol.90, pp.337-348.2004

23. S. Sultornsanee, I. Zeid, S. Kamarthi," Classification of Electromyogram Using Recurrence Quantification Analysis “, Complex Adaptive Systems, Vol 1,

24. Cihan H. Dagli, Editor in Chief Conference Organized by Missouri University of Science and Technology,2011Chicago, IL.

25. K. Marri and R. Swaminathan: 'Fatigue Analysis of Triceps Brachii Muscle using sEMG Signals and Recurrence Quantification Technique', Journal of Life Sciences and Technologies Vol. 4, No. 2, December 2016
This work is licensed under Creative Commons Attribution 4.0 License

To Submit Your Article Click Here: Submit Manuscript

DOI: $10.31579 / 2641-0419 / 185$
Ready to submit your research? Choose Auctores and benefit from:

*ast, convenient online submission
rigorous peer review by experienced research in your field
rapid publication on acceptance
* authors retain copyrights
* unique DOI for all articles
immediate, unrestricted online access

At Auctores, research is always in progress.

Learn more www.auctoresonline.org/journals/clinical-cardiology-andcardiovascular-interventions 